\title{
Generation and Starch Characterization of Non-Transgenic BEl and BEllb Double Mutant Rice (Oryza sativa) with Ultra-High Level of Resistant Starch
}

Satoko Miura, Nana Koyama, Naoko Crofts, Yuko Hosaka, Misato Abe and Naoko Fujita * (D)

\begin{abstract}
Background: Cereals high in resistant starch (RS) are gaining popularity, as their intake is thought to help manage diabetes and prediabetes. Number of patients suffering from diabetes is also increasing in Asian countries where people consume rice as a staple food, hence generation of practically growable high RS rice line has been anticipated. It is known that suppression of starch branching enzyme (BE) Ilb increases RS content in cereals. To further increase RS content and for more practical use, we generated a non-transgenic be 1 bezb double mutant rice (Oryza sativa) line, which completely lacked both proteins, by crossing a be1 mutant with a be2b mutant.

Results: The be1 be2b mutant showed a decrease in intermediate amylopectin chains and an increase in long amylopectin chains compared with be2b. The amylose content of bel be2b mutant (51.7\%) was the highest among all pre-existing non-transgenic rice lines. To understand the effects of chewing cooked rice and cooking rice flour on RS content, RS content of mashed and un-mashed cooked rice as well as raw and gelatinized rice flour were measured using be 1 be $2 b$ and its parent mutant lines. The RS contents of mashed cooked rice and raw rice flour of be 1 be $2 b$ mutant ( $28.4 \%$ and $35.1 \%$, respectively) were 3 -fold higher than those of be2b mutant. Gel-filtration analyses of starch treated with digestive enzymes showed that the RS in bel bezb mutant was composed of the degradation products of amylose and long amylopectin chains. Seed weight of be 1 be $2 b$ mutant was approximately $60 \%$ of the wild type and rather heavier than that of be2b mutant.
\end{abstract}

Conclusions: The endosperm starch in be 1 be $2 b$ double mutant rice were enriched with long amylopectin chains. This led to a great increase in RS content in cooked rice grains and rice flour in bel be2b compared with be2b single mutant. be1 be2b generated in this study must serve as a good material for an ultra-high RS rice cultivar.

Keywords: Resistant starch, Rice (Oryza sativa), Starch branching enzyme, Endosperm starch, Amylopectin, Amylose

\section{Background}

Starch is produced from photosynthetic products through biological reactions and serves as an important carbohydrate source for heterotrophs. Starch is utilized not only in food industries but also in technological and medical industries, and the applications of starch are expected to expand further in future. Starch is of two

* Correspondence: naokof@akita-pu.ac.jp

Department of Biological Production, Akita Prefectural University, Akita 010-0195, Japan

Springer Open types: one type is easily degraded by digestive enzymes, while the other type, known as resistant starch (RS), is less susceptible to digestive enzymes, travels to the colon as a high molecular weight of partially digested starch, and functions similar to fiber (Englyst et al. 1992; Nugent 2005; Matsuki 2010). RS is further categorized into five classes (RS1-5), depending on its characteristics: RS1 is physically inaccessible to digestive enzymes because of thick cell walls; RS2 shows B-type crystal structure, based on the X-ray diffraction pattern; RS3 is

(c) The Author(s). 2020 Open Access This article is licensed under a Creative Commons Attribution 4.0 International License, which permits use, sharing, adaptation, distribution and reproduction in any medium or format, as long as you give appropriate credit to the original author(s) and the source, provide a link to the Creative Commons licence, and indicate if changes were made. The images or other third party material in this article are included in the article's Creative Commons licence, unless indicated otherwise in a credit line to the material. If material is not included in the article's Creative Commons licence and your intended use is not permitted by statutory regulation or exceeds the permitted use, you will need to obtain permission directly from the copyright holder. To view a copy of this licence, visit http://creativecommons.org/licenses/by/4.0/. 
retrograded starch, which is initially gelatinized and then recrystallized to form a stable structure; RS4 is chemically modified by esterification or etherification to reduce digestibility; and RS5 are starch granules that have been modified with lipids forming a starch -lipid complex (Englyst et al. 1992; Brown et al. 1995; Hasjim et al. 2010; Matsuki 2010). Intake of foods high in RS content suppresses an acute increase in blood sugar level and insulin response, and prolongs fullness (Nugent 2005; Matsuki 2010). Suppression of insulin response likely promotes fat burn, thus preventing lifestyle-related diseases such as diabetes (Wilcox 2005).

Amylopectin is a branched glucose polymer that represents the major component of starch. Branches of amylopectin are generated by starch branching enzymes (BEs) (Nishi et al. 2001; Nakamura 2002; Satoh et al. 2003b). Three BE isozymes exist in rice (Oryza sativa) and maize (Zea mays): BEI, BEIIa, and BEIIb. Among these isozymes, BEIIb play a major role in amylopectin branching and is strongly expressed in endosperm and kernels (Boyer and Preiss 1978; Dang and Boyer 1988; Ohdan et al. 2005). Single mutants of BEI, BEIIa, and BEIIb gene have been isolated in rice (Nishi et al. 2001; Nakamura 2002; Satoh et al. 2003a; Satoh et al. 2003b) and maize (Boyer and Preiss 1978; Blauth et al. 2001; Blauth et al. 2002). Rice $b e 2 b$ single mutant plants produce opaque seeds that weigh less than wild-type (WT) seeds (Nishi et al. 2001; Matsushima et al. 2012). Amylopectin chain length distribution analyses show that the $b e 2 b$ single mutants produce significantly less short amylopectin chains, with degree of polymerization (DP) $=$ 6-12 (Nishi et al. 2001; Asai et al. 2014; Nakata et al. 2018); therefore, BEIIb is thought to produce branch points of $A$ and $B_{1}$ chains of amylopectin. The reduction of starch synthase I (SSI) activity is accompanied by the loss of BEIIb in rice (Nishi et al. 2001). The be2b mutant exhibits a reduction in amylopectin synthesis and consequently an increase in the proportion of amylose compared with the WT (Asai et al. 2014). In addition, the gelatinization temperature of starch is higher in the $b e 2 b$ mutant because of the increase in intermediate amylopectin chains with DP $=14-24$ (Tanaka et al. 2004).

By contrast, the rice $b e 2 a$ mutant shows no significant difference in seed morphology and amylopectin chain length distribution compared with the WT (Nakamura 2002). Analysis of the recombinant BEIIa enzyme suggests that the function of BEIIa partially overlaps with or is complemented by BEI and/or BEIIb (Nakamura 2002). On the other hand, rice be1 mutant shows a slight decrease in long amylopectin chains $(\mathrm{DP}>37)$ and intermediate chains (DP $=12-21)$, and a slight increase in short amylopectin chains $(\mathrm{DP}<10)$, suggesting that $\mathrm{BEI}$ is involved in the generation of branch points of $B_{1}, B_{2}$, and $B_{3}$ chains (Satoh et al. 2003b). However, loss of BEI causes a very small change in amylose content, although the gelatinization temperature of be1 is lower than that of the WT (Satoh et al. 2003b; Abe et al. 2013; Abe et al. 2014).

Introduction of the rice BEIIb gene into the $b e 2 b$ null mutant of rice produced a variety of lines with different $B E I I b$ expression levels and diverse endosperm starch properties (Tanaka et al. 2004). Low expression of $B E I I b$ results in less amylopectin short chains $(\mathrm{DP}<16)$ (Tanaka et al. 2004). On the other hand, overexpression of BEIIb leads to the accumulation of excessively branched, water-soluble glucans enriched in amylopectin short chains $(\mathrm{DP}<16)$ (Tanaka et al. 2004). Taken together, these data show that the loss of BEIIb greatly affects starch properties, and the function of BEIIb cannot be compensated for by other BEs (BEI and BEIIa), indicating that BEIIb is indispensable. Furthermore, the loss of BEIIb drastically elevates RS content to much higher orders of magnitude than the RS content of high amylose rice cultivars such as indica rice and the $s s 3 a$ mutant (Zhou et al. 2016). This indicates that the increase in amylopectin long chains leads to a greater increase in the RS content than that caused by the increase in amylose content (Tsuiki et al. 2016).

The maize $b e 2 b$ mutant (amylomaize) has higher amylose content than the rice $b e 2 b$ mutant (Boyer et al. 1976; Nishi et al. 2001). Remarkably, the maize GEMS0067 lines, which was developed using germplasm obtained from USDA-ARS Germplasm Enhancement of Maize (GEM) project (Li et al. 2008), show extraordinarily high values of many parameters: apparent amylose content $=83.1-85.6 \%$; final gelatinization temperature $=$ $122.0-130.0^{\circ} \mathrm{C}$; RS content $=30.9-34.3 \%$. The maize GEMS-0067 lines are thought to be $b e 1$ be $2 b$ double mutants (Personal communication with Prof. Jane) (Li et al. 2008). The expression of BEI and BEIIb has been down-regulated in rice (Zhu et al. 2012; Wang et al. 2017; Pan et al. 2018; Sawada et al. 2018), barley (Hordeum vulgare) (Carciofi et al. 2012), and wheat (Triticum aestivum) (Regina et al. 2006). In indica rice, downregulation of $B E I$ and $B E I I b$ genes resulted in higher apparent contents of amylose (64.8\%) and RS (14.6\%) than the WT (Zhu et al. 2012). Simultaneous knockdown of multiple $B E$ genes likely suppressed amylopectin biosynthesis, which in turn enhanced amylose synthesis. In addition, the ratio of long amylopectin branches was also higher. Amylose was preferentially degraded in germinating seeds compared with the long amylopectin chains (Zhu et al. 2012; Wang et al. 2017; Pan et al. 2018). In japonica rice, down-regulation of both $B E I$ and $B E I I b$ genes resulted in much lower seed weight, with fewer intermediate chains ( $\mathrm{DP}=11-22)$, compared with the down-regulation of BEIIb alone (Sawada et al. 2018). In transgenic barley, suppression of all three $B E s$ resulted in amylose as the only endosperm starch and a very high RS content of gelatinized starch 
(65\%) (Carciofi et al. 2012). In transgenic wheat, suppression of BEIIb alone did not affect the amylose content; however, suppression of both BEIIa and BEIIb genes resulted in high amylose content (>70\%) (Regina et al. 2006). These studies suggest that suppression of multiple $\mathrm{BE}$ isozymes, rather than BEIIb alone, results in less amylopectin short chains and consequently higher amylose and RS contents.

Increasing the RS content of cereals is receiving considerable attention; however, factors that affect the digestion of starch upon intake, such as whether the cereals were cooked/raw and mashed/un-mashed, need to be studied further. The structure and composition of native starch, such as amylose content and branch length of amylopectin, have been analyzed in high RS cereals; however, the structure of residual glucans after treatment with digestive enzymes remains largely unknown. To determine the type of glucan structure that is beneficial for gastrointestinal health, it is important to understand the structure of RS and residual glucan.

In this study, we generated a non-transgenic be 1 be $2 b$ double mutant rice line, which completely lacked both BEI and BEIIb proteins. The structure of endosperm starch in the double mutant was analyzed to examine its relationship with the RS content of raw/gelatinized rice flour and mashed/un-mashed cooked rice grains. Furthermore, structures of RS in raw rice flour and mashed cooked rice prepared from high RS rice lines, $b e 2 b$ and be1 $b e 2 b$, were analyzed, and the RS structure was discussed.

\section{Results}

Absence of BEI and BEllb

Total proteins were extracted from the be1 be $2 b$ double mutant (\#1403), its parental single mutants be1 (EM557) and be2b (EM10), and WT cultivars (Taichung 65 and Kinmaze), and BEI and BEIIb proteins were detected by western blotting (Fig. 1). When BEI antibody was used, BEI signal was detected in WT cultivars and be $2 b$ single mutant, but not in be1 single mutant and be1 be $2 b$ double mutant (Fig. 1). Similarly, when BEIIb antibody was used, BEIIb signal was detected in WT cultivars and be1 single mutant but not in $b e 2 b$ and $b e 1$ be $2 b$ (Fig. 1). This clearly showed that the mutation sites in $b e 1$ and $b e 2 b$ (see Plant materials) lead to the deficiencies of corresponding proteins.

\section{Seed Morphology and Seed Weight}

Seeds of WT cultivars and be1 single mutant were translucent, while those of the $b e 2 b$ single mutant and $b e 1$ $b e 2 b$ double mutant were opaque (Fig. 2a, b). The weight of be1 seeds was $19.5 \mathrm{mg}$, which was similar to that of WT seeds (Taichung 65, 21.4 mg). On the other hand, the seed weight of $b e 2 b$ was $56.9 \%(11.1 \mathrm{mg})$ of the WT (Kinmaze), and that of the be1 be2b double mutant (12.2 mg) was $62.6 \%$ and $57.0 \%$ of Kinmaze and Taichung 65 , respectively, which were regarded as WT. The seed weight of $b e 1$ be $2 b$ was significantly heavier than that of be $2 b$ (Fig. 2).

Fertility rate of the double mutant, its parental mutants and the WTs for the past 3 years were calculated

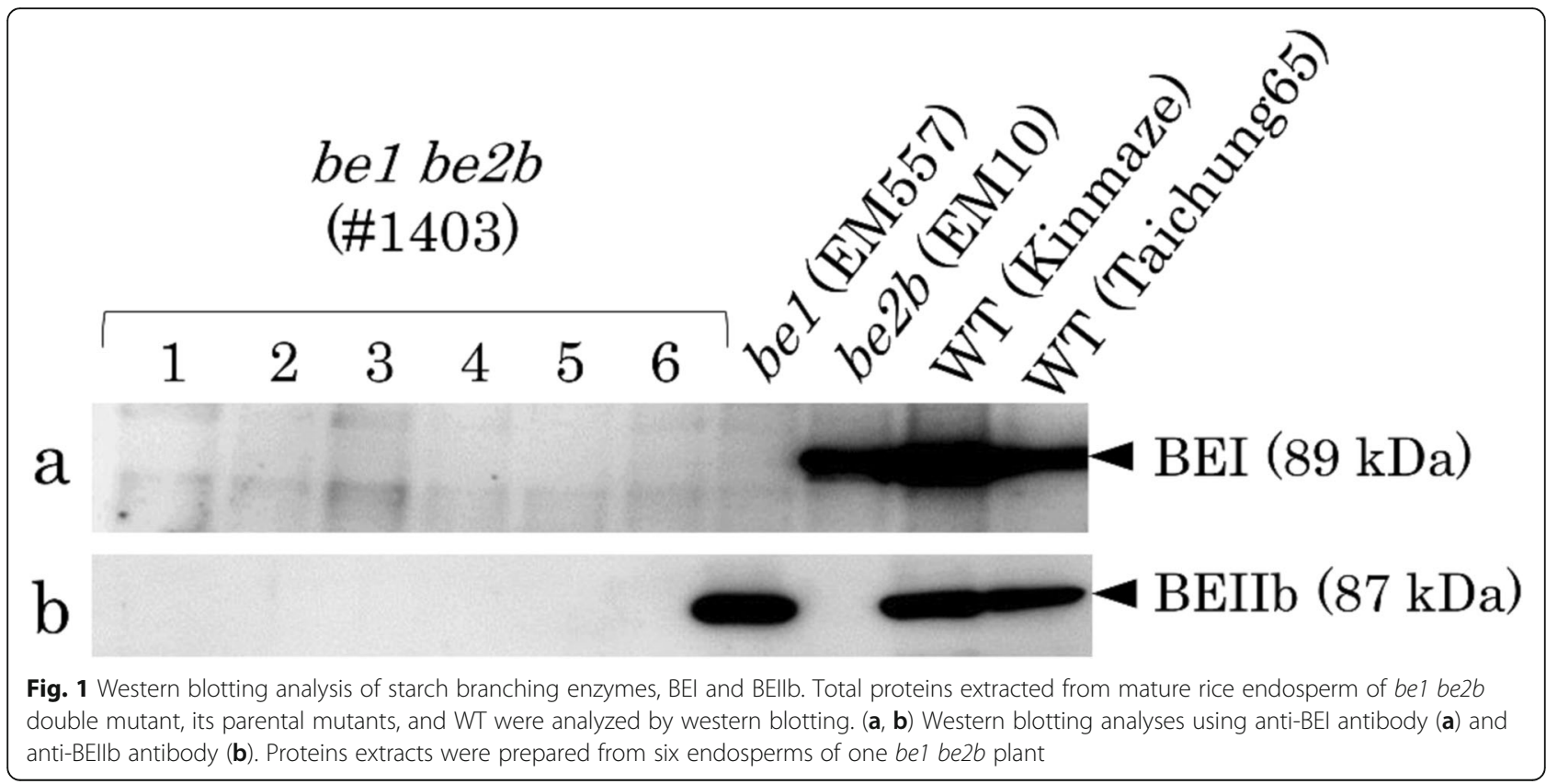




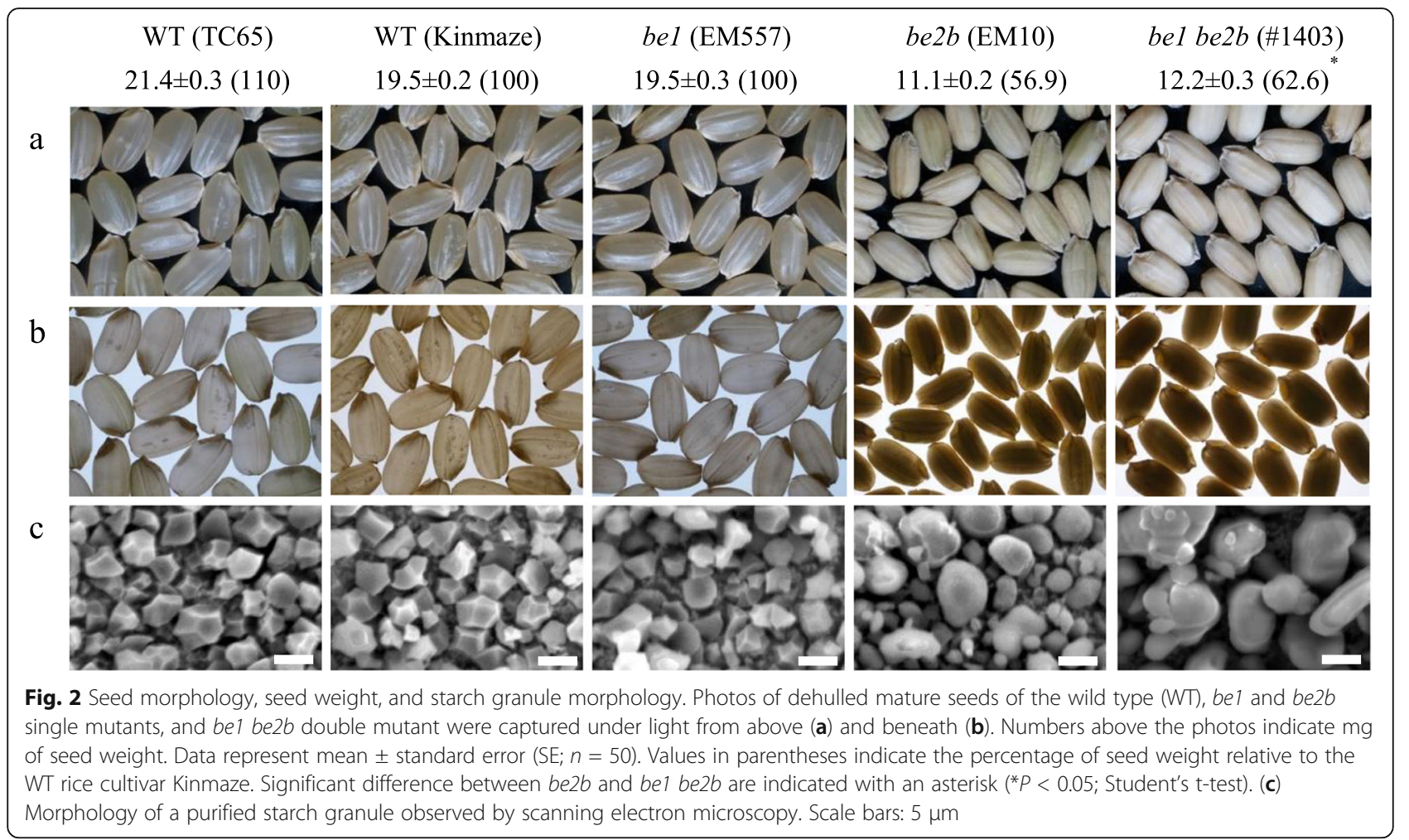

(Table 1). There were no significant differences among the lines in 2017. However, the fertility rate of the be1 single mutant were statistically lower than the WT Kinmaze in 2018 and Taichung 65 in 2019. While there were no significant differences in fertility rate between $b e 1$ be $2 b$ and other lines. In addition, growth of the plants was similar (Fig. 3), and there were no significant differences in number of panicles among the analyzed lines (Table 1). Length of culm tended to be dependent on the cultivar; Kinmaze and $b e 2 b$ were short, while Taichung 65 and be1 were long. Culm length of be1 be $2 b$ was similar to that of Kinmaze and $b e 2 b$. Therefore, the growth of be1 be $2 b$ was not impeded by the absence of two BE enzymes.

\section{Morphology, Crystallinity, and Thermal Properties of Starch Granules}

Starch granule morphology, crystallinity, and thermal properties were analyzed using purified starch granules. The starch granules of WT cultivars and bel single mutant observed by SEM were polygonal in shape and $2-5 \mu \mathrm{m}$ in size (Fig. 2c). By contrast, be $2 b$ and be 1 be $2 b$ mutants showed larger irregularly shaped starch granules that formed aggregates within the amyloplast (Fig. 2c; Asai et al. 2014). Peanut-like rod-shaped starch granules were also observed in $b e 1$ be $2 b$. High amylose content lines, such as rice $s s 3 a$ be $2 b$ and maize $b e 1$ be $2 b$ double mutants, also possess irregular rod-shaped starch granules, indicating that irregularly shaped starch granules

Table 1 Fertility rate for 3 years and panicle numbers and culm length of plants

\begin{tabular}{|c|c|c|c|c|c|}
\hline \multirow[t]{2}{*}{ Genotype } & \multicolumn{3}{|c|}{ Fertility rate $(\%)^{a}$} & \multirow{2}{*}{$\begin{array}{l}\text { Panicle } \\
\text { number }^{b}\end{array}$} & \multirow{2}{*}{$\begin{array}{l}\text { Culm } \\
\text { length }^{b}\end{array}$} \\
\hline & 2017 & '2018 & '2019 & & \\
\hline WT (Taichung 65) & $93.5 \pm 1.5 \mathrm{c}$ & $88.5 \pm 0.4 \mathrm{~cd}$ & $93.0 \pm 1.3 c$ & $17.4 \pm 1.0 \mathrm{~cd}$ & $81.2 \pm 1.0 c$ \\
\hline WT (Kinmaze) & $96.0 \pm 0.2 c$ & $90.5 \pm 1.3 c$ & $87.8 \pm 0.7 \mathrm{~cd}$ & $18.6 \pm 1.1 \mathrm{~cd}$ & $57.8 \pm 0.9 e$ \\
\hline bel (EM557) & $85.1 \pm 2.3 c$ & $71.7 \pm 0.9 d$ & $79.0 \pm 0.6 d$ & $16.2 \pm 1.2 d$ & $82.5 \pm 1.4 c$ \\
\hline be2b (EM10) & $89.0 \pm 2.4 c$ & $80.0 \pm 4.9 \mathrm{~cd}$ & $87.9 \pm 1.6 \mathrm{~cd}$ & $22.0 \pm 0.9 c$ & $67.8 \pm 1.2 \mathrm{~d}$ \\
\hline bel e2b (\#1403) & $96.1 \pm 0.3 c$ & $90.1 \pm 2.0 \mathrm{~cd}$ & $86.1 \pm 2.6 \mathrm{~cd}$ & $19.9 \pm 0.7 \mathrm{~cd}$ & $71.4 \pm 1.7 d$ \\
\hline
\end{tabular}

Data represent mean \pm standard error $\left(n=3^{\mathrm{a}}, n=10^{\mathrm{b}}\right)$

Different lowercase letters $(c-e)$ indicate significant differences among rice genotypes $(P<0.05$; Tukey-Kramer method) 


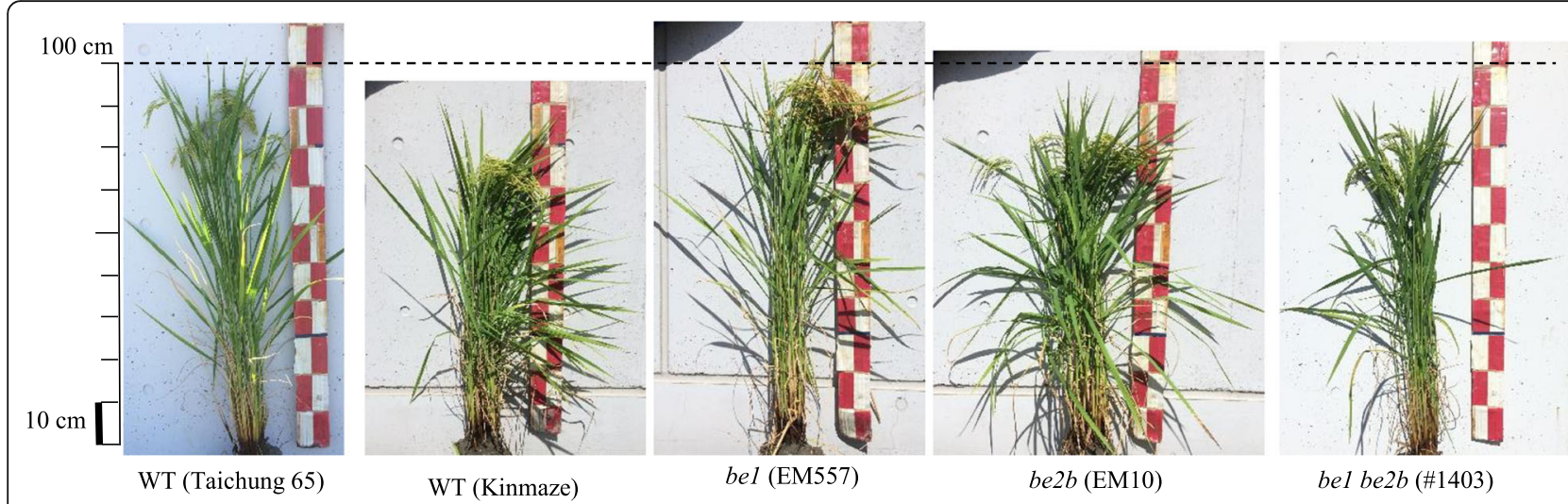

Fig. 3 Photos of typical rice plants at developing stage. Bars indicate $10 \mathrm{~cm}$

may be one of the characteristic features of lines with high amylose content (Jiang et al. 2010; Wei et al. 2010; Zhu et al. 2012; Asai et al. 2014).

Analysis of crystallinity of purified starch granules by Xray diffraction measurements revealed that starch granules of WT cultivars and be1 single mutant showed a typical A-type crystal structure, while those of $b e 2 b$ and $b e 1 b e 2 b$ showed a typical B-type crystal structure (Fig. 4). To calculate the relative starch crystallinity (RSC), calcium fluoride $\left(\mathrm{CaF}_{2}\right)$ was added as an internal standard $\left(2 \theta=28.3^{\circ}\right)$. There were no significant differences in RSC among rice lines by Tukey-Kramer method $(P<0.05)$ (Fig. 4).
The thermal properties of starch were analyzed by DSC (Table 2). The onset, peak, and conclusion gelatinization temperatures of $b e 1$ were $5.4{ }^{\circ} \mathrm{C}, 4.2{ }^{\circ} \mathrm{C}$, and $3.2{ }^{\circ} \mathrm{C}$ lower, respectively, than those of the parental WT cultivar Taichung 65; by contrast, the corresponding values of $b e 2 b$ were $6.8^{\circ} \mathrm{C}, 16.3^{\circ} \mathrm{C}$, and $23.8^{\circ} \mathrm{C}$ higher, respectively, than those of the parental WT cultivar Kinmaze. These values are consistent with previous studies (Satoh et al. 2003b; Tanaka et al. 2004). Strikingly, the onset, peak, and conclusion gelatinization temperatures of $b e 1$ be2b were $9.2^{\circ} \mathrm{C}, 7.5^{\circ} \mathrm{C}$, and $2.2^{\circ} \mathrm{C}$ higher, respectively, than those of $b e 2 b$. The required thermal energy of be1 $\left(10.0 \mathrm{~mJ} \mathrm{mg}^{-1}\right)$

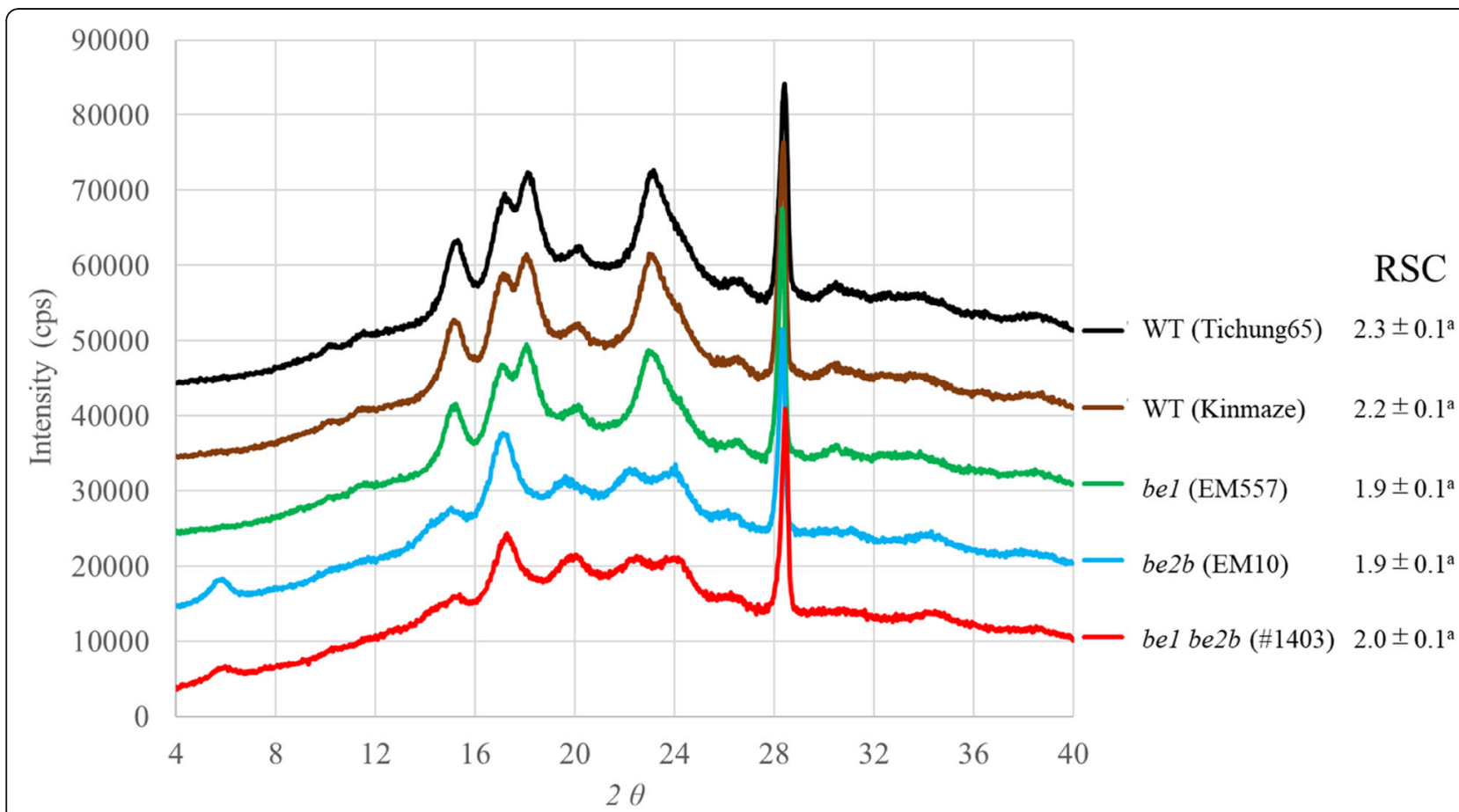

Fig. 4 Typical X-ray diffraction patterns and their relative starch crystallinity (RSC) of starch. Starch isolated from endosperm in WT cultivars, be1, be2b, and bel be2b were analyzed. There is no significant difference in RSC among rice lines by Tukey-Kramer method $(P<0.05)$ 
Table 2 Thermal properties of endosperm starch in different rice genotypes determined by differential scanning calorimetry (DSC)

\begin{tabular}{lllll}
\hline Genotype & \multicolumn{4}{l}{ Thermal properties } \\
\cline { 2 - 5 } & $\mathbf{T}_{\boldsymbol{o}}\left({ }^{\circ} \mathbf{C}\right)$ & $\mathbf{T}_{\boldsymbol{p}}\left({ }^{\circ} \mathbf{C}\right)$ & $\mathbf{T}_{\boldsymbol{c}}\left({ }^{\circ} \mathbf{C}\right)$ & $\Delta \mathbf{H}\left(\mathbf{m} \mathbf{~ m g}^{-\mathbf{1}}\right)$ \\
\hline WT (Taichung 65) & $51.0 \pm 0.1 \mathrm{~b}$ & $58.0 \pm 0.0 \mathrm{C}$ & $64.1 \pm 0.1 \mathrm{~b}$ & $11.3 \pm 0.1 \mathrm{ab}$ \\
WT (Kinmaze) & $45.4 \pm 0.0 \mathrm{c}$ & $53.0 \pm 0.1 \mathrm{~d}$ & $59.7 \pm 0.0 \mathrm{~b}$ & $9.6 \pm 0.2 \mathrm{~b}$ \\
be1 (EM557) & $45.6 \pm 0.3 \mathrm{C}$ & $53.8 \pm 0.1 \mathrm{~d}$ & $60.9 \pm 0.1 \mathrm{~b}$ & $10.0 \pm 0.4 \mathrm{~b}$ \\
be2b (EM10) & $52.2 \pm 0.6 \mathrm{~b}$ & $69.3 \pm 0.4 \mathrm{~b}$ & $83.5 \pm 1.6 \mathrm{a}$ & $17.6 \pm 2.0 \mathrm{a}$ \\
be1 be2b (\#1403) & $61.4 \pm 1.3 \mathrm{a}$ & $76.8 \pm 0.2 \mathrm{a}$ & $85.7 \pm 0.7 \mathrm{a}$ & $7.1 \pm 0.6 \mathrm{~b}$
\end{tabular}

Data represent mean \pm standard error $(n=3)$. Different lowercase letters $(a-d)$ indicate significant differences among rice genotypes $(P<0.05$; Tukey-Kramer method). To, onset temperature; $T p$, peak temperature; Tc, conclusion temperature; $\Delta \mathrm{H}$, gelatinization enthalpy of starch was slightly less than that of Taichung $65\left(11.3 \mathrm{~mJ} \mathrm{mg}^{-1}\right)$, whereas that of $b e 2 b\left(17.6 \mathrm{~mJ} \mathrm{mg}^{-1}\right)$ was considerably greater than that of Kinmaze $\left(9.6 \mathrm{~mJ} \mathrm{mg}^{-1}\right)$. By contrast, the required thermal energy of $b e 1$ be $2 b\left(7.1 \mathrm{~mJ} \mathrm{mg}^{-1}\right)$ was much less than that of $b e 2 b$ (Table 2), possibly because the amylose content of $b e 1$ be $2 b$ was higher than that of $b e 2 b$ (described below).

\section{Analyses of Starch Structure}

To determine the basis of differences observed in the crystallinity and gelatinization temperatures between be1 $b e 2 b$, parental mutant lines, and the WT, we analyzed the chain length distribution of amylopectin by capillary electrophoresis (Fig. 5). The chain length distribution patterns
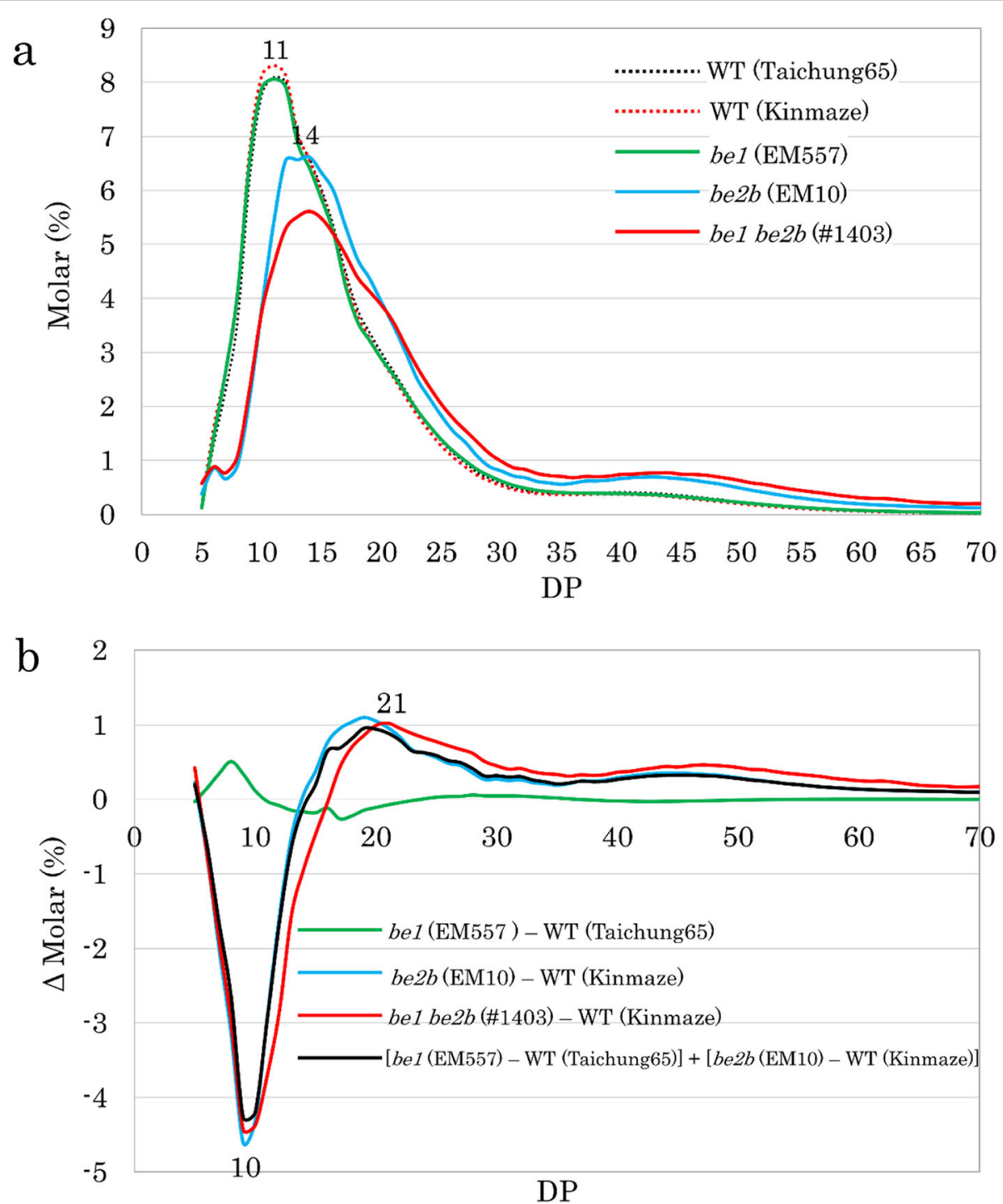

Fig. 5 Chain length distribution of endosperm amylopectin analyzed by capillary electrophoresis. a Chain length distribution patterns of WT, be1, be2b, and be1 be2b. $\mathbf{b}$ Differences in the fine structure of amylopectin between the WT and mutant lines. Differences are shown as $\Delta$ Molar \%, and the value was calculated by subtracting the pattern of WT from each mutant line, as indicated. Black line indicates a theoretical value calculated by adding the effects of the loss of BEl alone and BEllb alone 
of Taichung 65, Kinmaze, and be1 were similar to each other, with a peak at DP 11 (Fig. 5a). On the other hand, $b e 2 b$ and $b e 1$ be $2 b$ showed a peak at DP 14 and also a broader peak at approximately DP 44, although the peak at DP 14 was lower and that at DP 44 was higher in be1 $b e 2 b$ that those in $b e 2 b$ (Fig. 5a). To compare the detailed differences in chain length distribution patterns, the patterns of WT cultivars were subtracted from those of mutant lines (Fig. 5b). The subtraction curve of be1 (minus Taichung 65) showed an increase in DP 6-10 and DP 2436, and a minor decrease in DP 37-54 (Fig. 5b), consistent with a previous study (Satoh et al. 2003b). The subtraction curve of $b e 2 b$ (minus Kinmaze) showed a considerable decrease in DP 6-13 and an increase in DP > 14 with broad peaks at DP 19 and DP 45 (Fig. 5b), consistent with previous studies (Nishi et al. 2001; Abe et al. 2014; Asai et al. 2014). The subtraction curve of be1 be $2 b$ (minus Kinmaze) also showed a great decrease in DP 6-16 and an increase in DP $>17$, with broad peaks at DP 21 and DP 47 (Fig. 5b). When the subtraction curves of be1 be2b and $b e 2 b$ (minus Kinmaze) were compared, that of $b e 1$ be $2 b$ at DP $>10$ shifted toward the longer side. Notably, be 1 be $2 b$ showed less chains with DP 10-20 but more chains with DP $>21$ than $b e 2 b$ (Fig. 5b).

To determine the apparent amylose content, true amylose content, and the extra-long chain content, and the ratio of long- to short-chains of amylopectin, we analyzed the debranched purified starch and isolated amylopectin by gel filtration chromatography (Fig. S1); the percentages are summarized in Table 3. The apparent amylose content (fraction I of starch) includes true amylose content and extra-long chains of amylopectin. Fraction II contains long-chains, and fraction III contains short-chains of amylopectin (Fig. S1) (Horibata et al. 2004). The apparent amylose contents of Taichung 65, Kinmaze, and be1 were approximately $20 \%$, while that of $b e 2 b$ was significantly higher than that of the WT by $26.5 \%$, consistent with previous studies (Abe et al. 2014; Asai et al. 2014). Strikingly, the apparent amylose content of be1 be $2 b$ was 51.7\%, which was almost double that of $b e 2 b$. The content of extra-long chains of amylopectin in $b e 1$ be $2 b(1.3 \%)$ was less than in other lines including Taichung 65 (1.8\%), Kinmaze (2.5\%), be1 (2.0\%), and $b e 2 b(2.0 \%)$ (Table 3). Therefore, the true amylose content of $b e 1$ be $2 b$ was extremely high (50.5\%). The ratio of short- to long-chain of amylopectin (fraction III/II) was approximately 3.0 (starch or amylopectin) in Taichung 65, Kinmaze, and be1; however, this value was 1.0 (starch) or 0.9 (amylopectin) in $b e 2 b$ and 0.5 (starch) or 0.6 (amylopectin) in be1 be2b, indicating that the content of long-chains of amylopectin was the highest in $b e 1$ be $2 b$, followed by $b e 2 b$.

\section{RS Content and Structure}

Since $b e 1$ be $2 b$ accumulated high levels of amylose and long-chains of amylopectin, further experiments were conducted to analyze the content and structure of RS in various rice genotypes. RS contents were measured using raw and gelatinized rice flour as well as mashed and unmashed cooked rice (Table 4). The content of RS was low in raw flour prepared from WT cultivars (Taichung 65 and Kinmaze) and be1 single mutant $(0.2 \%$ and $0.1 \%$, respectively) but considerably higher in raw flour prepared from $b e 2 b$ and $b e 1$ be $2 b$ (11.6\% and $35.1 \%$, respectively). The RS contents of gelatinized rice flour prepared from WT cultivars and be1 were $0.9 \%$ and $0.5 \%$, respectively, which were slightly higher than the values obtained from the raw rice flour of these genotypes. In $b e 2 b$, the RS content of gelatinized rice flour was one-third $(3.9 \%)$ of that of raw rice flour, whereas in be1 $b e 2 b$, the RS content of gelatinized rice flour was three quarters $(26.6 \%)$ of that of raw rice flour. The content of RS in un-mashed cooked rice prepared from Taichung 65, Kinmaze, and be1 was low (1.3\%, 1.2\%, and $1.4 \%$, respectively) but considerably higher in un-mashed cooked rice prepared from $b e 2 b$ and $b e 1$ be $2 b$ (27.5\% and $76.2 \%$, respectively). The RS content of $b e 2 b$ (EM10) in un-mashed cooked rice in the previous report (Itoh et al. 2017) was $21.6 \%$, which was lower than this study

Table 3 Apparent and true amylose contents and short/long amylopectin branch ratios of starch and amylopectin

\begin{tabular}{|c|c|c|c|c|c|}
\hline \multirow[t]{2}{*}{ Genotype } & \multicolumn{2}{|c|}{ Fraction I (\%) ${ }^{a}$} & \multicolumn{2}{|c|}{ III/II ratio ${ }^{b}$} & \multirow{2}{*}{$\begin{array}{l}\text { TAC (\%) } \\
\text { Starch }\end{array}$} \\
\hline & $\begin{array}{l}\text { Starch } \\
\text { (AAC) }\end{array}$ & $\begin{array}{l}\text { Amylopectin } \\
\text { (ELC) }\end{array}$ & Starch & Amylopectin & \\
\hline WT (Taichung 65) & $21.1 \pm 0.3 f$ & $1.8 \pm 0.1 \mathrm{de}$ & $3.0 \pm 0.0 \mathrm{~d}$ & $3.1 \pm 0.1 d$ & 19.2 \\
\hline WT (Kinmaze) & $21.6 \pm 2.0 f$ & $2.5 \pm 0.2 d$ & $2.8 \pm 0.2 d$ & $3.2 \pm 0.1 d$ & 19.1 \\
\hline bel (EM557) & $21.6 \pm 0.3 f$ & $2.0 \pm 0.1 d$ & $3.2 \pm 0.0 \mathrm{~d}$ & $3.3 \pm 0.1 d$ & 19.5 \\
\hline be2b (EM10) & $26.5 \pm 0.7 e$ & $2.0 \pm 0.1 d$ & $1.0 \pm 0.0 \mathrm{e}$ & $0.9 \pm 0.1 \mathrm{e}$ & 24.5 \\
\hline bel be2b (\#1403) & $51.7 \pm 0.4 d$ & $1.3 \pm 0.1 \mathrm{e}$ & $0.5 \pm 0.0 f$ & $0.6 \pm 0.0 \mathrm{e}$ & 50.5 \\
\hline
\end{tabular}

${ }^{a}$ Fraction I of starch analyzed by gel filtration chromatography represents the apparent amylose content (AAC), and that of purified amylopectin represents the extra-long chain content (ELC)

${ }^{b}$ III/II ratio represents the ratio of amylopectin short chains (fraction III) to amylopectin long chains (Fraction II). Data represent mean \pm SE ( $n=3$ ). Each fraction was split as shown in Fig. S1

'True amylose content (TAC) was calculated by subtracting ELC from AAC

Different lowercase letters $(\mathrm{d}-\mathrm{f})$ indicate significant differences among rice genotypes $(P<0.05$; Tukey-Kramer method) 
Table 4 RS contents of raw and gelatinized rice flour and mashed and un-mashed cooked rice

\begin{tabular}{lllll}
\hline Genotype & Raw rice flour & Gelatinized rice flour & Un-mashed cooked rice & Mashed cooked rice \\
\hline WT (Taichung 65) & $0.2 \pm 0.0 \mathrm{c}$ & $0.9 \pm 0.0 \mathrm{c}$ & $1.3 \pm 0.1 \mathrm{c}$ & $0.9 \pm 0.0 \mathrm{c}$ \\
WT (Kinmaze) & $0.2 \pm 0.0 \mathrm{c}$ & $0.9 \pm 0.0 \mathrm{c}$ & $1.2 \pm 0.0 \mathrm{c}$ & $0.9 \pm 0.0 \mathrm{c}$ \\
bel (EM557) & $0.1 \pm 0.0 \mathrm{c}$ & $0.5 \pm 0.0 \mathrm{c}$ & $1.4 \pm 0.3 \mathrm{c}$ & $1.2 \pm 0.0 \mathrm{c}$ \\
be2b (EM10) & $11.6 \pm 0.2 \mathrm{~b}$ & $3.9 \pm 0.0 \mathrm{~b}$ & $27.5 \pm 1.6 \mathrm{~b}$ & $10.8 \pm 0.2 \mathrm{~b}$ \\
bel be2b (\#1403) & $35.1 \pm 1.0 \mathrm{a}$ & $26.6 \pm 0.2 \mathrm{a}$ & $76.2 \pm 1.4 \mathrm{a}$ & $28.4 \pm 0.4 \mathrm{a}$ \\
\hline
\end{tabular}

Data represent mean \pm SE $(n=3)$. Different lowercase letters $(a-c)$ indicate significant differences among rice genotypes $(P<0.05$; Tukey-Kramer method)

(Table 4). This difference may be caused by the difference in amount of water added to steam rice (water was 1.8 and 1.5 folds of the weight of polished rice in previous report and current study, respectively). The RS contents of mashed cooked rice prepared from WT cultivars (Taichung 65 and Kinmaze) and be1 were $0.9 \%$ and $1.2 \%$, respectively, which were slightly lower than those of un-mashed cooked rice. By contrast, the RS contents of mashed cooked rice prepared from $b e 2 b$ and $b e 1$ be $2 b$ were $10.8 \%$ and $28.4 \%$, respectively, which were reduced to approximately $40 \%$ compared with the RS contents of un-mashed cooked rice.

To determine RS structure, RS was purified from raw rice flour and mashed cooked rice of high RS lines, $b e 2 b$ and $b e 1$ be $2 b$. The RS samples were solubilized in DMSO, debranched, analyzed by gel filtration chromatography, and compared with the elution profile of purified starch (Fig. 6). In $b e 2 b$, the elution profile of purified starch showed peak amylose, amylopectin long chains, and amylopectin short chains at 107, 146, and $166 \mathrm{~min}$, respectively (Fig. 6). Although the same three peaks were observed in the elution profile of RS obtained from raw rice flour of $b e 2 b$, the first peak of RS was negligible compared with that of purified starch; by contrast, the second and third peaks of RS (obtained at 144 and $160 \mathrm{~min}$, respectively) were higher than those of purified starch, with a shift toward higher molecular weight. In the elution profile of RS obtained from mashed cooked rice of $b e 2 b$, the first peak was slightly higher than that of RS from raw rice flour but much lower than that of purified starch. The elution profile of RS obtained from mashed cooked rice of $b e 2 b$ did not show a clear second peak but rather a broad elution pattern. The third peak of RS obtained from mashed cooked rice of $b e 2 b$ was higher than that of purified starch but lower than that of RS from raw rice flour, and was obtained at a higher molecular weight at $158 \mathrm{~min}$. In contrast to $b e 2 b$, the elution pattern of $b e 1$ be $2 b$ purified starch showed a very high amylose peak at $107 \mathrm{~min}$, broad peak at 120-140 min, low peak of amylopectin long chains at $144 \mathrm{~min}$, and very low peak of amylopectin short chains at $160 \mathrm{~min}$. The elution profile of RS obtained from $b e 1$ be $2 b$ raw rice flour showed three peaks; the area of the first peak was $30-40 \%$ of the area of purified starch, while the second and third peaks were higher than those of purified starch. The elution profile of RS obtained from be1 be $2 b$ mashed cooked rice showed only two peaks; the first peak was considerably reduced compared with that of purified starch, while the second peak (at $146 \mathrm{~min}$ ) was high and appeared to be amalgamated.

\section{Discussion}

\section{Generation of Non-transgenic Rice Lines Deficient in BEI} and BEllb

Rice is one of the top three crops with the highest production in the world, and serves as the major dietary source of carbohydrates in Asian and African countries (Fairhurst and Dobermann 2002; Awika 2011). Given the recent increase in the number of diabetic patients worldwide, new rice lines allowing the restriction of carbohydrate intake are being developed (Wang et al. 2017). Among the various starch biosynthetic enzymes, it is clear that reduction in the level of amylopectin biosynthesis enzymes, BEIIb and/or SSIIIa, leads to high amylose content (Fujita 2014; Wang et al. 2017). Importantly, suppression of multiple BE isozymes allows the accumulation of amylose and RS to high levels in maize (Li et al. 2008), wheat (Regina et al. 2006; Corrado et al. 2020), barley (Regina et al. 2010; Carciofi et al. 2012), and rice (described later). In a rat feeding trial, intake of RS improved gastrointestinal health (Regina et al. 2006; Zhu et al. 2012), and prevented sudden increase in blood glucose levels (Zhu et al. 2012). In rice, the expression of $B E I$ and BEIIb genes has been suppressed using antisense RNA (Wei et al. 2010), artificial microRNA (Butardo et al. 2011), and RNA interference (RNAi) technology (Sawada et al. 2018), and the resulting transgenic lines showed drastic starch phenotypes compared with the WT. However, there are no information about the seed weight or agricultural traits in these transgenic rice lines, except that the materials produced by RNAi technology, which suppressing BEI and BEIIb, significantly reduced it seed weight (Sawada et al. 2018). Unlike these previous studies, we generated a nontransgenic be 1 be $2 b$ double mutant rice line for more practical use by crossing single mutant lines that are completely deficient in BEI or BEIIb. Therefore, the effects of loss of these enzymes are more prominent compared with the previously generated transgenic rice lines 

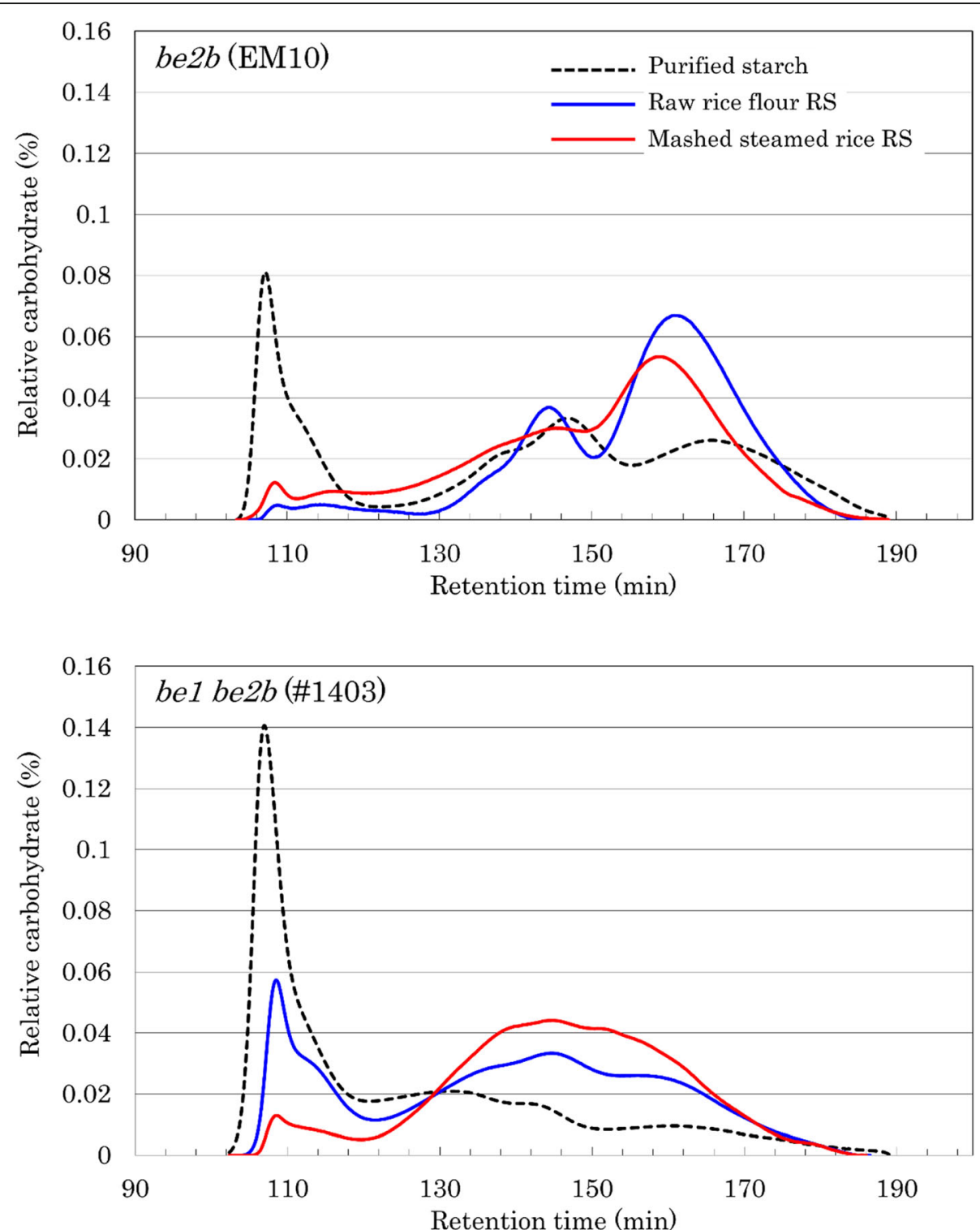

Fig. 6 Structure of resistant starch (RS) in raw rice flour and mashed cooked rice. RS samples prepared from be2b and bel be2b were debranched by isoamylase treatment and analyzed by gel filtration chromatography. The difference in retention time (RT) to Fig. S1 was due to different lot of the column

which have low levels of these enzymes, although it cannot be compared directly due to the difference of the methods.

The be1 be2b double mutant showed higher seed weight than the $b e 2 b$ single mutant (Fig. 2) over three consecutive years $(2016,2017$, and 2018; data not shown). There are at least three reasons why the seed weight of $b e 1$ be $2 b$ was heavier than that of $b e 2 b$ single mutant. The first possibility is that the absence of both BEI and BEIIb diminished amylopectin biosynthesis but enhanced amylose synthesis. Loss of BEIIb is accompanied by the reduction in SSI activity to $50 \%$, which may be due to the absence of branches that serve as primers for SSI. SSI activity in $b e 1$ be $2 b$ was also lower than that in the WT (Miura et al., in preparation). Less amylopectin branches in $b e 1 b e 2 b$ result in less non-reducing ends to serve as a substrate for SS. This leads to less consumption of ADP-glucose by SS isozymes including SSI. Then, an abundance of ADP-glucose in amyloplasts enhances amylose biosynthesis by granule-bound starch synthase I (GBSSI). The second possibility is that be1 $b e 2 b$ may have inherited some seed weight traits from Taichung 65. The seed weight of Taichung 65, the parental WT genotype of be1, was slightly (1.1-fold) heavier than that of Kinmaze, the parental WT genotype of $b e 2 b$ (Fig. 2), and this tendency was observed in all 3 years 
(2016, 2017, and 2018; data not shown). The third possibility is the difference in flowering time. Plants of $b e 2 b$ (Kinmaze background) flowered in late August to early September, whereas those of be1 (Taichung 65 background) and be 1 be $2 b$ flowered in mid-August in Akita, Japan. Therefore, it is possible that the be1 be $2 b$ double mutant inherited flowering loci from be1 (Taichung 65 background). Earlier flowering time guarantees higher temperature during the seed development, which results in higher activity of starch biosynthetic enzymes, thus enhancing starch synthesis.

In addition, the growth (Fig. 3) and fertility rate of be 1 be $2 b$ plants (Table 1 ) was similar to those of the WT. These data suggest that the presence of BEIIa alone is sufficient for plant growth and the accumulation of up to $60 \%$ endosperm starch compared with the WT. The agricultural traits of $b e 1 b e 2 b$ such as seed weight and growth are beneficial for commercializing the double mutant as an ultra-high RS rice cultivar, although further breeding such as a backcrossing with elite rice cultivars is necessary. Back-crossing of be 1 $b e 2 b$ mutant to the high yield elite rice cultivar is currently being conducted and its offspring is selected using molecular makers generated against the mutation sites. It is hoped that the back-crossed lines will be used widely as practical high RS rice cultivars in near future.

\section{Effects of Loss of both BEI and BEllb on Amylopectin Structure and Thermal Properties}

It is clear that the loss of BEIIb, among the three BE isozymes, drastically affects starch properties by decreasing amylopectin short chains (Nishi et al. 2001; Nakamura 2002; Takahashi and Fujita 2017). The be1 be2b double mutant synthesized less amylopectin chains with DP 1020 and more amylopectin chains with DP > 20 than the be $2 b$ single mutant (Fig. $5 \mathrm{a}$ and $\mathrm{b}$ ), resulting in a higher ratio of long to short amylopectin branches (Table 3). These results are consistent with the GEMS-0067 lines in maize, which are thought to be be1 be2b double mutants (Li et al. 2008), and with the down-regulation of $B E I$ and BEIIb genes in transgenic rice (Zhu et al. 2012; Sawada et al. 2018; Lin et al. 2019). Comparison of the theoretical and actual subtraction curves of chain length distribution showed that the theoretical curve obtained by the addition of the subtraction curves of $b e 1$ and $b e 2 b$ single mutants $[(b e 1-\mathrm{WT})+(b e 2 b-\mathrm{WT})]$ were less chains with DP $10-20$ and more chains with DP $\geq$ 40 (Fig. 5b) than that of the actual curve of $b e 1$ be $2 b$ (be1 be $2 b-\mathrm{WT})$. This indicates a synergistic effect due to the loss of both $\mathrm{BE}$ isozymes, suggesting that in the absence of BEIIb, the remaining BE enzymes (BEI and/ or BEIIa) may generate amylopectin branches with DP 10-20; however, when both BEI and BEIIb are absent,
BEIIa alone may not be able to compensate for their absence, and the number of branches with DP 10-20 may also be diminished. The average and median values of amylopectin chain length distribution (Table S1) were DP 26-27 and DP 14, respectively, in WT cultivars, and DP 33.8 and DP 17 in $b e 2 b$; however, both values were greater in be1 be $2 b$ (average, DP 36.7; median, DP 19), suggesting that amylopectin possessed extremely long double-helical branch chains in the absence of BEI and BEIIb.

These unique starch structures greatly influence the gelatinization temperature. High temperature and energy are required to unwind double-helical structure (Hizukuri 1986); therefore, the longer the amylopectin branches, the higher the gelatinization temperature. Most of double helices in amylopectin molecules consist of amylopectin branches with $\mathrm{DP} \leq 24$ within a cluster of amylopectin. The greater the number of short amylopectin chains $(\mathrm{DP} \leq 12)$ and the lower the number of intermediate chains $(13 \leq \mathrm{DP} \leq 24)$, the lower the gelatinization temperature (Fujita 2014; Hayashi et al. 2015). The increase in the gelatinization temperature of be $2 b$ by $16^{\circ} \mathrm{C}$ compared with the WT may be because $b e 2 b$ showed a significant decrease in amylopectin with $\mathrm{DP} \leq 12$ and a great increase in amylopectin with DP 13-24 (Table S1). Although be1 be2b had slightly less branches with $\mathrm{DP} \leq 12$ compared with $b e 2 b$, branches with DP $13-24$ in $b e 1$ be $2 b$ were significantly less than those in $b e 2 b$ (Table S1). However, the peak gelatinization temperature of $b e 1$ be $2 b$ was significantly higher than that of $b e 2 b$ by $7.5^{\circ} \mathrm{C}$. The amylopectin chain length responsible for gelatinization temperature is essentially $\mathrm{DP} \leq 24$ in the WT as described above. However, formation of even longer double helices in $b e 2 b$ (EM10) and be1 be $2 b$ likely resulted in an extremely high gelatinization temperature due to the significant increase in DP 25-36 in be1 be $2 b$ compared with that of $b e 2 b$ (Table S1). These trends are in agreement with the results of previous studies on maize GEMS-0067 lines ( $\mathrm{Li}$ et al. 2008) and transgenic rice lines down-regulated for the expression of BEI and BEIIb genes (Lin et al. 2019).

\section{Effects of Loss of both BEI and BEllb on Amylose Content}

The apparent amylose content of be1 be $2 b$ was $51.7 \%$, as measured by gel filtration chromatography. This value was higher than the current highest record of $45.1 \%$ in the non-transgenic ss $3 a$ be $2 b$ double mutant japonica rice (\#4019) (Asai et al. 2014). The amylose content of a transgenic indica rice line, in which the expression of $B E I$ and BEIIb was down-regulated, was $44.8 \%$, based on gel filtration chromatography (Zhu et al. 2012), while that of maize GEMS-0067 lines was 83.1-85.6\%, based on the iodine method ( $\mathrm{Li}$ et al. 2008). Suppression of 
both $B E I$ and $B E I I b$ genes leads to higher amylose content compared with the suppression of BEIIb alone, which is in agreement with previous studies ( $\mathrm{Li}$ et al. 2008; Wang et al. 2018). In these cases, loss of two major branching enzymes, BEI and BEIIb, involved in amylopectin biosynthesis likely resulted in enhanced amylose biosynthesis.

Rice cultivars with high amylose content can be divided into two types: high true amylose content cultivars and high extra-long amylopectin chain content cultivars (Horibata et al. 2004). Extra-long chain is a long linear glucan, similar to amylose but connected to amylopectin, synthesized by GBSSI which is encoded by Waxy (Wx) gene (Takeda et al. 1987). The difference in extra-long chain content is thought to be dependent on single nucleotide polymorphisms (SNPs) in the Wx gene (Crofts et al. 2019). The be1 be $2 b$ double mutant used in this study was low in extra-long chain (1.3\%) (Table 3, Fig. S1) but high in true amylose content (50.5\%). This was because be1 be $2 b$ harbors the $W x^{b}\left(g b s s 1^{L}\right)$ gene; this gene was inherited from either Kinmaze or Taichung 65 and contains a thymine instead of a guanine at the first nucleotide position of intron 1, resulting in a low level of GBSSI protein (Crofts et al. 2019).

The maize GEMS-0067 lines accumulate higher levels of amylose and intermediate components than the amylose-extender (ae) lines, in which intermediate components, i.e., glucan molecules, are smaller than normal amylopectin but are enriched with longer average branch chains (Li et al. 2008). The elution profile of debranched purified starch obtained by gel filtration chromatography showed that amylose in be1 be $2 b$ was eluted as a broad peak at 110-120 min, which did not return completely to the baseline, unlike that observed in $b e 2 b$ (Fig. S1). On the other hand, the elution profile of purified amylopectin in be1 be $2 b$ showed almost no peak around 110 min, indicating that glucans eluted from the purified starch at approximately $110-120 \mathrm{~min}$ may be low molecular weight amylose. Thus, starch in the rice be 1 be $2 b$ double mutant may have some different characteristics from the intermediate chains in maize GEMS-0067 lines. The apparent amylose content (83-86\%) and extra-long chain content (20-26.8\%) of maize GEMS-0067 lines were much higher than those of rice $b e 1 b e 2 b$, as measured by the gel filtration method (Li et al. 2008). Furthermore, suppression of all three genes encoding BE isozymes (BEI, BEIIa, and BEIIb) in transgenic barley resulted in a further increase in amylose content (99.1\%), as measured by the iodine method (Carciofi et al. 2012). Considering these cases, amylose content of be1 be $2 b$ was relatively low; however, this is because $b e 1$ be $2 b$ harbors the $W^{b}$ gene that encodes low levels of GBSSI (Sano 1984), whereas maize and barley lines harbor the normal $W x$ gene, which encodes high levels of GBSSI.
On the other hand, when both BEI and BEIIb activities were suppressed in transgenic indica rice harboring the $W x^{a}$ gene, which encodes high levels of GBSSI, the amylose content was $64.8 \%$ as determined by the iodine method (Zhu et al. 2012). Although the amylose content measured by different methods cannot be directly compared, regardless of the level of GBSSI, rice $b e 1 b e 2 b$ including previous and our studies seems to contain lower amylose content than other plant species. This suggests that additional factors prevent excess biosynthesis or accumulation of amylose in rice.

\section{Effects of Loss of both BEI and BEllb on RS Content}

Amylopectin, the major component of starch, forms double helices using adjacent branches in its native state. When starch absorbs water and gets heated, it undergoes gelatinization. The double helices are then unwound, and gelatinized starch is easily broken down by digestive enzymes. When the gelatinized starch is cooled, nearby branches re-form double helices to produce retrograded starch, although those double helices may be imperfect. When the amylopectin branch chains are long, starch is harder to gelatinize, quicker to retrograde, and therefore less degradable. By contrast, when the amylopectin branch chains are short, starch is easier to gelatinize, slower to retrograde, and therefore more degradable. Previously, we showed that the loss of BEIIb increases the amount of long amylopectin chains, which form long double helices, leading to a significant increase in RS content compared with high amylose rice lines such as indica rice and SSIIIa deficient mutant rice (Tsuiki et al. 2016; Zhou et al. 2016).

In this study, the $b e 2 b$ single mutant showed significantly higher RS content than WT cultivars and be1, regardless of the product type and the method of preparation (raw or gelatinized rice flour and mashed or un-mashed cooked rice). Strikingly, the be1 be $2 b$ double mutant showed 2.6-6.8-fold higher RS content than $b e 2 b$ (Table 4), possibly because of the high apparent amylose content as well as high amylopectin long branches in be1 be $2 b$. This means that the amounts of be 1 be $2 b$ required to produce a food product with a certain level of RS content is only one-third to seventh of the $b e 2 b$. These findings in non-transgenic japonica rice, be 1 be $2 b$, are consistent with those in maize GEMS0067 lines (Jiang et al. 2010) as well as in transgenic japonica rice lines suppressed from the expression of $B E I$ and BEIIb genes (Lin et al. 2019).

Rice is utilized in many different food applications, in addition to the ordinary cooked rice. To reflect those differences in applications, the RS contents were analyzed using samples prepared by four different methods. Although chewing of foods greatly 
varies among individuals, the RS content of cooked rice would be intermediate between that of unmashed and completely mashed cooked rice. Additionally, although the degree of gelatinization may vary with the application, such as bread, cookies, and noodles, the RS content of rice flour would be intermediate between that of raw and completely gelatinized rice flour. Comparison of RS content of rice samples prepared by different methods revealed that the RS content of all analyzed rice lines was the highest in un-mashed cooked rice (Table 4). This was perhaps because the digestive enzymes could not function effectively in un-mashed cooked rice, given the small surface area, and this is thought to be a typical feature of RS1. In the WT and be1 mutant, the second highest RS content was detected in mashed cooked rice and gelatinized rice flour, while the lowest RS content was detected in raw rice flour (Table 4), possibly because the digestive enzymes functioned effectively in raw flour, given its large surface area. It has been suggested that the majority of starch in the raw rice flour can be digested by $\alpha$-amylase, even though it has A-type crystallinity. The reason why gelatinized rice flour showed higher RS content than raw rice flour may be because grinding with pestle and mortar after gelatinization resulted in the samples becoming sticky and slightly lumpy, leading to lower surface area for digestive enzymes. On the other hand, in $b e 2 b$ and $b e 1$ be $2 b$, the second highest RS content was detected in raw rice flour, followed by mashed cooked rice, and the least in gelatinized starch. The reason why raw rice flour showed higher RS content than mashed cooked rice and gelatinized rice flour may be explained by two possibilities. The loss of BEIIb in $b e 2 b$ and be1 be $2 b \mathrm{mu}-$ tants probably increased long amylopectin chains, which formed longer double helices with B-type crystallinity and were more resistant to degradation by digestive enzymes. The other possibility is that gelatinized rice flour and mashed cooked rice prepared from $b e 2 b$ and $b e 1$ be $2 b$ were rapidly retrograded; this is thought to be a typical feature of RS3. Although some lumps appeared during the grinding procedure, the lumps of $b e 2 b$ and $b e 1 b e 2 b$ were less sticky than that of the WT and be1 and could be easily suspended in the digestive enzyme solution; therefore, samples had a larger surface area for digestive enzymes to work effectively.

\section{Effects of Loss of both BEI and BEllb on the Structure of RS} Detailed analyses of RS structure have been performed only in maize GEMS-0067 and ae lines (Jiang et al. 2010). RS samples of GEMS-0067 lines treated with thermostable $\alpha$-amylase at $95-100{ }^{\circ} \mathrm{C}$ contained two components: high molecular weight glucans (DP 840951), including amylose and slightly branched glucans derived from intermediate components, and linear, low molecular weight glucans (DP 59-74) (Jiang et al. 2010). In this study, RS samples were prepared by treating rice samples with digestive enzymes at $37^{\circ} \mathrm{C}$. The remaining RS materials were debranched and analyzed by gel filtration chromatography (Fig. 6). The RS structure of $b e 2 b$ rice flour showed almost no amylose peak, while that of be1 $b e 2 b$ rice flour showed a clear amylose peak, although the peak area of $b e 1 b e 2 b$ RS from raw rice flour was $30-40 \%$ of the peak area of be1 be $2 b$ purified starch. This may be because $b e 1 b e 2 b$ has a very high true amylose content (50.5\%), and the amylose may have formed long double helices. Of the true amylose content, 30$40 \%$ could not be degraded by digestive enzymes and therefore maintained the original molecular weight of amylose, while $60 \%$ was partially degraded to lower molecular weight amylose; hence, the peaks at 145 and 160 min, corresponding to amylopectin long chains and amylopectin short chains, respectively, were higher than those of purified starch. It is also possible that the partially degraded amylose, which was of a similar molecular weight as the long amylopectin chains, formed double helices, thus avoiding degradation by digestive enzymes. The RS sample of $b e 1$ be $2 b$ rice flour showed a small peak at $160 \mathrm{~min}$. This peak is thought to represent the remaining long amylopectin chains within the size of one amylopectin cluster, and corresponds to RS2. The first peak of the RS structure of mashed cooked rice from $b e 1 b e 2 b$ was diminished similar to that of $b e 2 b$. This suggests that almost all gelatinized amylose can be degraded to lower molecular weight amylose. The peak at 120-130 min corresponding to low molecular weight amylose from purified starch was lower in RS prepared from mashed cooked rice of $b e 1$ be $2 b$ than those of purified starch. It can be speculated that the low molecular weight amylose can also be degraded to an even lower molecular weight. The RS prepared from mashed cooked rice of $b e 2 b$ showed a clear peak at $158 \mathrm{~min}$, which corresponds to long amylopectin chains within the size of one amylopectin cluster, as described above. By contrast, the RS prepared from mashed cooked rice of be1 be $2 b$ showed a large peak at 130-170 min, with the tallest peak at $144 \mathrm{~min}$. We speculate that this peak represents the degradation products of amylose, long amylopectin chains spanning two or more clusters, and chains within one cluster. The gelatinized starch was incubated with digestive enzymes at $37^{\circ} \mathrm{C}$. It is possible that starch retrograded and formed double helices with a variety of different sized glucans, thus transforming into RS3, which is more resistant to degradation by digestive enzymes. The type of glucan molecules that form double helices in raw rice flour and retrograded starch can only be speculated at this moment, and further detailed analyses of RS structure are required. In addition, the actual RS 
content and structure in processed foods should also be determined in future studies.

\section{Conclusion}

The be1 be $2 b$ rice mutant completely lacking both enzymes at the protein levels was generated. Seed weight of $b e 1$ be $2 b$ mutant was approximately $60 \%$ of the wild type and rather heavier than that of $b e 2 b$ mutant over the past three consecutive years. The $b e 1$ be $2 b$ mutant showed a decrease in intermediate amylopectin chains with DP10-20 and an increase in long amylopectin chains with DP $>21$ compared with $b e 2 b$. The amylose content of $b e 1$ be $2 b$ mutant (51.7\%) was the highest among all pre-existing non-transgenic rice lines. The RS contents of mashed cooked rice and raw rice flour of be 1 be $2 b$ mutant were 3-fold higher than those of $b e 2 b$ mutant. Gel-filtration analyses of starch treated with digestive enzymes showed that the RS in $b e 1$ be $2 b$ mutant was composed of the degradation products of amylose and long amylopectin chains. be1 be $2 b$ generated in this study must serve as a good material for an ultra-high RS rice cultivar that expected of lifestyle-related diseases such as diabetes all over the world.

\section{Methods}

\section{Plant Materials}

Rice (Oryza sativa L.) $b e 1$ and $b e 2 b$ single mutant lines, EM557(be1) (Satoh et al. 2003b) and EM10 (be2b) (Nishi et al. 2001), respectively, were previously isolated from $\mathrm{N}$-methyl-N-nitrosourea (NMU)-mutagenized populations of japonica WT cultivars of Taichung 65 and Kinmaze, respectively. be1 (EM557) possesses a single nucleotide mutation at the end of exon 10 of $B E I$ gene, which introduces a stop codon into exon 11, resulting in no BEI protein (unpublished data). $b e 2 b$ possesses a single nucleotide mutation at the end of intron 9 of BEIIb gene, which produces incorrect mRNA (EM10, Wada et al., in preparation). The $b e 1$ and $b e 2 b$ single mutants were crossed, and the $F_{2}$ population was screened. Opaque seeds were analyzed by western blotting (Fig. 1), and a double be 1 be $2 b$ mutant line, namely \#1403, were selected. $F_{4}$ and $F_{5}$ seeds were used for subsequent analysis. All rice lines were grown in a paddy field of Akita Prefectural University during the summer months under natural conditions.

\section{Western Blotting}

Single mature rice grains without embryo were ground to a fine powder. Total proteins were extracted using $300 \mu \mathrm{L}$ sample buffer containing $125 \mathrm{mM}$ Tris- $\mathrm{HCl}(\mathrm{pH}$ 6.8), $8 \mathrm{M}$ urea, $4 \%(\mathrm{w} / \mathrm{v})$ sodium dodecyl sulfate (SDS), $5 \%(\mathrm{v} / \mathrm{v}) \beta$-mercaptoethanol, and $0.05 \%(\mathrm{w} / \mathrm{v})$ bromophenol blue. Samples were continuously agitated for $3 \mathrm{~h}$, and then centrifuged at $15,000 \mathrm{rpm}(20,000 \mathrm{~g})$ at room temperature for 10 mins. The supernatant of each sample was loaded onto a $7.5 \%$ polyacrylamide gel and separated by SDS-polyacrylamide gel electrophoresis (PAGE). Proteins in the gel were blotted onto a polyvinylidene fluoride (PVDF) membrane and detected by anti-BEI (Satoh et al. 2003b) and anti-BEIIb (Nishi et al. 2001) antisera with 1:2000 and 1:5000 dilutions, respectively, as described previously (Crofts et al. 2012; Crofts et al. 2015).

\section{Seed Morphology}

The morphology of brown rice grains was observed as described previously (Miura et al. 2018). using a stereomicroscope (SZX7-ILST-0 (SP), OLYMPUS, Tokyo, Japan), with a light source from above and beneath. Photos were taken using a digital camera.

\section{Starch Analyses}

Purified starch was extracted from polished rice grains using the cold-alkaline method, as described previously (Yamamoto et al. 1973; Yamamoto et al. 1981). Purified starch granules were coated with gold using a fine coater (JEOL JFC-1200, Tokyo, Japan) for $120 \mathrm{~s}$. Starch granule morphology was observed by scanning electron microscopy (SEM; HITACHI TM3030Plus, Tokyo, Japan) in a secondary electron mode at $15 \mathrm{kV}$, according to the method of Fujita et al. (2003). To perform X-ray diffraction measurements, purified starch isolated using the above method was equilibrated in a chamber at room temperature and $100 \%$ relative humidity for $24 \mathrm{~h}$. The X-ray diffraction patterns of starch were measured using copper, nickel foil-filtered, $K \alpha$ radiation with the X-ray diffractometer (MiniFlex600 Rigaku, Tokyo, Japan) at $40 \mathrm{kV}$ and $20 \mathrm{~mA}$. The scanning region of two-theta angle $(2 \theta)$ varied from $5.0^{\circ}$ to $40.0^{\circ}$, with a scanning speed of $0.3^{\circ} \mathrm{s}^{-1}$ (Fujita et al. 2006). The relative starch crystallinity (RSC) of each endosperm starch from the mutant lines was determined by the method described in a previous report (Kodama et al. 2011; Abe et al. 2013) using calcium fluoride as an internal standard. The thermal properties of purified starch were determined by differential scanning calorimetry (DSC; Seiko Instrument 6100, Chiba, Japan) and analyzed as described previously (Fujita et al. 2003; Fujita et al. 2006).

\section{Starch Structure Analyses}

The chain length distribution of endosperm starch was determined by capillary electrophoresis (P/ACE MDQ Plus Carbohydrate System, AB Sciex, Framingham, Ma, USA), according to the method of Fujita et al. (2001). Amylopectin was isolated from $1 \mathrm{~g}$ purified starch using n-butanol and isoamyl alcohol, as described previously 
(Schoch 1957; Takeda et al. 1986). The purified starch and isolated amylopectin were debranched using Pseudomonas isoamylase (Hayashibara, Okayama, Japan) and analyzed by gel filtration chromatography (Toyopearl HW-55S and HW-50S $\times 3$, Tosoh, Tokyo, Japan) (Horibata et al. 2004; Fujita et al. 2007; Toyosawa et al. 2016). The apparent amylose content, ratio of short- to longchain amylopectin, true amylose content, and extralong-chain amylopectin content were calculated as described previously (Horibata et al. 2004; Fujita et al. 2007; Toyosawa et al. 2016).

\section{Measurement of RS Content}

RS contents were analyzed in cooked rice (mashed and un-mashed) and rice flour (raw and gelatinized). To prepare cooked rice, $1 \mathrm{~g}$ polished rice was washed twice with distilled water and cooked with 1.5 volumes of water (w/w of dry polished rice) in $15 \mathrm{~mL}$ plastic test tubes using a rice cooker (NS-WF10, Zojirushi, Osaka, Japan). Then, $250 \mathrm{mg}$ mashed and un-mashed cooked rice were transferred to new tubes. To prepare rice flour, polished rice was ground to a fine powder using a mortar and pestle, and sieved through a $100 \mu \mathrm{m}$ colander. For the raw rice flour test, $100 \mathrm{mg}$ rice flour was transferred to a tube. For the gelatinized rice flour test, 100 $\mathrm{mg}$ rice flour was boiled with two volumes of water $(\mathrm{w} /$ w) for 10 mins and the resulting rice pastes were ground again with a pestle and mortar. Samples were digested in $4 \mathrm{~mL}$ digestive enzymes using the RS assay kit (Megazyme, Bray, Ireland) containing porcine pancreatic $\alpha$ amylase $(120 \mathrm{U})$ and amyloglucosidase $(12 \mathrm{U})$ at $37^{\circ} \mathrm{C}$ for $16 \mathrm{~h}$ by continuous shaking. RS contents were measured as described previously (Tsuiki et al. 2016).

\section{Analysis of RS Structure}

Mashed cooked rice and raw rice flour (1 $\mathrm{g}$ each) were digested in $40 \mathrm{~mL}$ digestive enzyme solution, as described above, and precipitated with an equal volume of ethanol. Samples were then centrifuged at $3000 \mathrm{rpm}$ $(937 \mathrm{~g})$ at room temperature for 10 mins. The precipitate containing RS was washed with $40 \mathrm{~mL}$ of $50 \%$ ethanol. The precipitation and centrifugation steps were repeated once again. The RS pellet was dissolved in 100\% dimethyl sulfoxide (DMSO) and boiled for $1 \mathrm{~h}$, while shaking every 10 mins. The RS solution was centrifuged at $3000 \mathrm{rpm}(937 \mathrm{~g})$ at room temperature for $10 \mathrm{mins}$. The RS samples were precipitated by mixing with three volumes of $100 \%$ ethanol and incubated at $-30^{\circ} \mathrm{C}$ for $1 \mathrm{~h}$. The RS materials were centrifuged at $3000 \mathrm{rpm}(937 \mathrm{~g}$ ) at $4{ }^{\circ} \mathrm{C}$ for 10 mins, and the resulting pellet was suspended in $5 \mathrm{~mL}$ of $100 \%$ ethanol. After centrifugation at $3000 \mathrm{rpm}(937 \mathrm{~g})$ at $4{ }^{\circ} \mathrm{C}$ for $10 \mathrm{mins}$, the RS pellets were dried in a speed vacuum. The dried samples were ground to a fine powder, and $3-5 \mathrm{mg}$ of the powdered
RS material was gelatinized in $1 \mathrm{~N} \mathrm{NaOH}$ and neutralized with $1 \mathrm{~N} \mathrm{HCl}$. Then, the gelatinized RS was debranched using Pseudomonas isoamylase (Hayashibara, Okayama, Japan) to perform gel filtration analysis, as described above.

\section{Data Analyses}

DSC, carbohydrate contents of each fraction by gelfiltration chromatography, RS contents, RSC by X-ray diffraction and percentages and average of chain length of amylopectin by capillary electrophoresis data were statistically analyzed by a one-way variance (ANOVA) and Tukey-Kramer method $(p<0.05)$. Significant difference of seed weight between $b e 2 b$ and $b e 1$ be $2 b$ was tested by Student's t-test $(p<0.05)$.

\section{Supplementary Information}

The online version contains supplementary material available at https://doi. org/10.1186/s12284-020-00441-0.

Additional file 1: Table S1. Percentages and average chain length of amylopectin branches analyzed by capillary electrophoresis.

Additional file 2: Fig. S1. Elution profiles of debranched starch and amylopectin analyzed by gel filtration chromatography. Fraction I contains amylose or extra-long amylopectin chains. Fraction II contains long amylopectin chains. Fraction III contains short amylopectin chains. Red lines indicate patterns obtained from starch, and blue lines indicate patterns obtained from the purified amylopectin. Differences in retention time (RT) in Kinmaze were due to difference of the lot of the column.

\section{Acknowledgments \\ The authors thank Ms. Yuko Nakaizumi (Akita Prefectural University) for growing the rice plants and Bio-Edit for English language review. The authors also thank Prof. Hikaru Satoh for providing parental mutant lines, be 1 (EM557) and be2b (EM10). Pseudomonas isoamylase used for debranching amylopectin was a kind gift from Hayashibara Co., Ltd.}

\section{Authors' Contributions}

SM performed experiments, analyzed data and wrote the paper. NK performed preliminary experiments. NC isolated a double mutant and wrote the paper. YH helped the experiments. MA helped the isolation of double mutant. NF designed research, performed experiments and wrote the paper. The author(s) read and approved the final manuscript.

\section{Funding}

This work was supported by the Science and Technology Research Promotion Program for Agriculture, Forestry and Fisheries and Food Industry (25033AB and 28029C; NF), the President's Funds of Akita Prefectural University (N.F. and N.C.), Grant-in Aid for JSPS fellows from Japan Society for the Promotion of Science (\#15J40176 and JP18J40020; NC), and the Japan Society for the Promotion of Science (\#16K18571, JP18K14438, and 20 K05961; N.C. and 19H01608; N.F.).

Availability of Data and Materials Not applicable.

Ethics Approval and Consent to Participate Not applicable.

Consent for Publication

Not applicable.

Competing Interests

The authors declare that they have no competing interests. 
Received: 12 July 2020 Accepted: 23 November 2020

Published online: 06 January 2021

\section{References}

Abe N, Asai H, Yago H, Oitome FN, Itoh R, Crofts N, Nakamura Y, Fujita N (2014) Relationships between starch synthase I and branching enzyme isozymes determined using double mutant rice lines. BMC Plant Biol 14:80

Abe N, Nakamura Y, Fujita N (2013) Thermal properties, morphology of starch granules and crystallinity of endosperm starch in $\mathrm{SSI}$ and $\mathrm{BE}$ isozymes double mutant lines. J Appl Glycosci 60:171-176

Asai H, Abe N, Matsushima R, Crofts N, Oitome FN, Nakamura Y, Fujita N (2014) Deficiencies in both starch synthase Illa and branching enzyme llb lead to a significant increase in amylose in SSIlla-inactive japonica rice seeds. J Exp Bot 65:5497-5507

Awika JM (2011) Advances in cereal science: implications to food processing and health promotion. In: Awika J.M, Piironen V, Bean S (eds) American Chemical Society, ACS symposium series 1089. Washington DC, 1-13

Blauth SL, Kim K, Klucinec JD, Shannon JC, Thompson DB, Guiltinan MJ (2002) Identification of Mutator insertional mutants of starch-branching enzyme 1 (sbe1) in Zea mays L. Plant Mol Biol 48:287-297

Blauth SL, Yao Y, Klucinec JD, Shannon JC, Thompson DB, Guiltinan MJ (2001) Identification of Mutator insertional mutants of starch-branching enzyme 2a in corn. Plant Physiol 125:1396-1405

Boyer CD, Garwood DL, Shannon JC (1976) The interaction of the amyloseextender and waxy mutants of maize (Zea Mays L.) fine structure of amyloseextender waxy starch. Starch 28:405-410

Boyer CD, Preiss J (1978) Multiple forms of starch branching enzyme of maize: evidence for independent genetic control. Biochem Biophys Res Commun 80:169-175

Brown IL, McNaught KJ, Moloney E (1995) Hi-maize: new directions in starch technology and nutrition. Food Australia - Official J Cafta and AIFST 47:272-275

Butardo VM, Fitzgerald MA, Bird AR, Gidley MJ, Flanagan BM, Larroque O, Resurreccion AP, Laidlaw HK, Jobling SA, Morell MK, Rahman S (2011) Impact of down-regulation of starch branching enzyme llb in rice by artificial microRNA- and hairpin RNA-mediated RNA silencing. J Exp Bot 62:4927-4941

Carciofi M, Blennow A, Jensen SL, Shaik SS, Henriksen A, Buleon A, Holm PB, Hebelstrup KH (2012) Concerted suppression of all starch branching enzyme genes in barley produces amylose-only starch granules. BMC Plant Biol 12: 223

Corrado M, Cherta-Murillo A, Chambers ES, Wood AJ, Plummer A, Lovegrove A, Edwards CH, Frostb GS, Hazard BA (2020) Effect of semolina pudding prepared from starch branching enzyme lla and $b$ mutant wheat on glycaemic response in vitro and in vivo: a randomised controlled pilot study. Food Funct 11:617-627

Crofts N, Abe K, Aihara S, Itoh R, Nakamura Y, Itoh K, Fujita N (2012) Lack of starch synthase Illa and high expression of granule-bound starch synthase I synergistically increase the apparent amylose content in rice endosperm. Plant Sci 193-194:62-69

Crofts N, Abe N, Oitome NF, Matsushima R, Hayashi M, Tetlow IJ, Emes MJ, Nakamura Y, Fujita N (2015) Amylopectin biosynthetic enzymes from developing rice seed form enzymatically active protein complexes. J Exp Bot 66:4469-4482

Crofts N, Itoh A, Abe M, Miura S, Oitome FN, Bao J, Fujita N (2019) Three major nucleotide polymorphisms in the waxy gene correlated with the amounts of extra-long chains of amylopectin in rice cultivars with $\mathrm{S}$ or L-type amylopectin nucleotide polymorphisms in waxy genes responsible for extralong chain content in rice. J Appl Glycosci 66:37-46

Dang PL, Boyer CD (1988) Maize leaf and kernel starch synthases and starch branching enzymes. Phytochem 27:1255-1259

Englyst HN, Kingman SM, Cummings JH (1992) Classification and measurement of nutritionally important starch fractions. Eur J Clin Nutr 46:33-50

Fairhurst TH, Dobermann A (2002) Rice in the global food supply. Better Crops Int 16:3-6

Fujita N (2014) Starch biosynthesis in rice endosperm. AGri-Bioscience Monographs 4:1-18

Fujita N, Hasegawa H, Taira T (2001) The isolation and charavterization of waxy mutant of diploid wheat (Triticum monococcum L.). Plant Sci 160:595-602

Fujita N, Kubo A, Suh D, Wong K, Jane J, Ozawa K, Takaiwa F, Inaba Y, Nakamura $Y$ (2003) Antisense inhibition of isoamylase alters the structure of amylopectin and the physicochemical properties of starch in rice endosperm. Plant Cell Physiol 44:607-618
Fujita N, Yoshida M, Asakura N, Ohdan T, Miyao A, Hirochika H, Nakamura Y (2006) Function and characterization of starch synthase I using mutants in rice. Plant Physiol 140:1070-1084

Fujita N, Yoshida M, Kondo T, Saito K, Utsumi Y, Tokunaga Y, Nishi A, Satoh H, Park JH, Jane JL, Miyao A, Hirochika H, Nakamura Y (2007) Characterization of SSIIla-deficient mutants of rice: the function of SSIIla and pleiotropic effects by SSIlla deficiency in the rice endosperm. Plant Physiol 144:2009-2023

Hasjim J, Lee S, Hendrich S, Setiawan S, Ai Y, Jane J (2010) Characterization of a novel resistant-starch and its effects on postprandial plasma-glucose and insulin responses. Cereal Chem 87:257-262

Hayashi M, Kodama M, Nakamura Y, Fujita N (2015) Thermal and pasting properties, morphology of starch granules, and crystallinity of endosperm starch in the rice SSI and SSIlla double-mutant. J Appl Glycosci 62:81-86

Hizukuri S (1986) Polymodal distribution of the chain lengths of amylopectins, and its significance. Carbohydr Res 147:342-347

Horibata T, Nakamoto M, Fuwa H, Inouchi N (2004) Structural and physicochemical characteristics of endosperm starches of rice cultivars recently bred in Japan. J Appl Glycosci 51:303-313

Itoh Y, Crofts N, Abe M, Hosaka Y, Fujita N (2017) Characterization of the endosperm starch and the pleiotropic effects of biosynthetic enzymes on their properties in novel mutant rice lines with high resistant starch and amylose content. Plant Sci 258:52-60

Jiang H, Campbellb M, Blancoc M, Jane J (2010) Characterization of maize amylose-extender (ae) mutant starches: part II. Structures and properties of starch residues remaining after enzymatic hydrolysis at boiling-water temperature. Carbohydr Polym 80:1-12

Kodama I, Shibata C, Fujita N, Ishikawa K, Takahashi T, Nakamura Y, Kawamoto T, Kato K, Sato K, Matsunami M, Akiyama Y (2011) Starch properties of waxy rice cultivars influencing rice cake hardening. Japan J Food Engineering 12(4): 157-162

Li L, Jiang H, Campbell M, Blanco M, Jane J (2008) Characterization of maize amylose-extender (ae) mutant starches. Part l: relationship between resistant starch contents and molecular structures. Carbohydr Polym 74:396-404

Lin L, Pan T, Liu Q, Wei C (2019) Cooking, morphological, mechanical and digestion properties of cooked rice with suppression of starch branching enzymes. Int J Biol Macromol 137:187-196

Matsuki J (2010) Resistant starch. J Jpn Soc Food Sci 57:224

Matsushima R, Yamashita J, Kariyama S, Enomoto T, Sakamoto W (2012) A phylogenetic re-evaluation of morphological variations of starch grains among Poaceae species. J Appl Glycosci 60:37-44

Miura S, Crofts N, Saito Y, Hosaka Y, Oitome NF, Watanabe T, Kumamaru T, Fujita N (2018) Starch synthase lla-deficient mutant rice line produces endosperm starch with lower gelatinization temperature than japonica rice cultivars. Front Plant Sci 15:645

Nakamura Y (2002) Towards a better understanding of the metabolic system for amylopectin biosynthesis in plants: rice endosperm as a model tissue. Plant Cell Physiol 43:718-725

Nakata M, Miyashita T, Kimura R, Nakata Y, Takagi H, Kuroda M, Yamaguchi T, Umemoto T, Yamakawa H (2018) MutMapPlus identified novel mutant alleles of a rice starch branching enzyme llb gene for fine-tuning of cooked rice texture. Plant Biotechnol J 16:111-123

Nishi A, Nakamura Y, Tanaka N, Satoh H (2001) Biochemical and genetic analysis of the effects of amylase-extender mutation in rice endosperm. Plant Physiol 127:459-472

Nugent P (2005) Health properties of resistant starch. Nutr Bull 30:27-54

Ohdan T, Francisco PB Jr, Sawada T, Hirose T, Terao T, Satoh H, Nakamura Y (2005) Expression profiling of genes involved in starch synthesis in sink and source organs of rice. J Exp Bot 56:3229-3244

Pan T, Lin L, Wang J, Liu Q, Wei C (2018) Long branch-chains of amylopectin with B-type crystallinity in rice seed with inhibition of starch branching enzyme I and IIb resist in situ degradation and inhibit plant growth during seedling development: degradation of rice starch with inhibition of SBE//IIb during seedling development. BMC Plant Biol 18:9

Regina A, Bird A, Topping D, Bowden S, Freeman J, Barsby T, Kosar-Hashemi B, Li Z, Rahman S, Morell M (2006) High-amylose wheat generated by RNA interference improves indices of large-bowel health in rats. PNAS 103:3546-3551

Regina A, Kosar-Hashemi B, Ling S, Li Z, Rahman S, Morell M (2010) Control of starch branching in barley defined through differential RNAi suppression of starch branching enzyme lla and Ilb. J Exp Bot 61:1469-1482

Sano $Y(1984)$ Differential regulation of waxy gene expression in rice endosperm. Theor Appl Genet 68:467-473 
Satoh H, Nishi A, Fujita N, Kubo A, Nakamura Y, Kawasaki T, Okita TW (2003a) Isolation and characterization of starch mutants in rice. J Appl Glycosci 50: 225-230

Satoh H, Nishi A, Yamashita K, Takemoto Y, Tanaka Y, Hosaka Y, Aya S, Fujita N, Nakamura $Y$ (2003b) Starch-branching enzyme I-deficient mutation specifically affects the structure and properties of starch in rice endosperm. Plant Physiol 133:1111-1121

Sawada T, Itoh M, Nakamura Y (2018) Contributions of three starch branching enzyme Isozymes to the fine structure of amylopectin in rice endosperm. Front Plant Sci 9:1536

Schoch TJ (1957) Preparation of starch and the starch fractions. Methods Enzymol 3:5-17

Takahashi T, Fujita N (2017) Thermal and rheological characteristics of mutant rice starches with widespread variation of amylose content and amylopectin structure. Hydrocol 62:83-93

Takeda Y, Hizukuri S, Juliano BO (1986) Purification and structure of amylose from rice starch. Carbohydr Res 148(2):299-308

Takeda Y, Hizukuri S, Takeda C, Suzuki A (1987) Structures of branched molecules of amyloses of various origins, and molar fractions of branched and unbranched molecules. Carbohydr Res 165(1):139-145

Tanaka N, Fujita N, Nishi A, Satoh H, Hosaka Y, Ugaki M, Kawasaki S, Nakamura Y (2004) The structure of starch can be manipulated by changing the expression levels of starch branching enzyme $\mathrm{llb}$ in rice endosperm. Plant Biotechnol J 2:507-516

Toyosawa Y, Kawagoe Y, Matsushima R, Crofts N, Ogawa M, Fukuda M, Kumamaru T, Okazaki Y, Kusano M, Saito K, Toyooka K, Sato M, Ai Y, Jane J L, Nakamura Y, Fujita N (2016) Deficiency of starch synthase Illa and IVb alters starch granule morphology from polyhedral to spherical in rice endosperm. Plant Physiol 170:1255-1270

Tsuiki K, Fujisawa H, Itoh A, Sato M, Fujita N (2016) Alterations of starch structure lead to increased resistant starch of steamed rice: identification of high resistant starch rice line. J Cereal Sci 68:88-92

Wang J, Hu P, Chen Z, Liu Q, Wei C (2017) Progress in high-amylose cereal crops through inactivation of starch branching enzymes. Front Plant Sci 8:469

Wang J, Hu P, Lin L, Chen Z, Liu Q, Wei C (2018) Gradually decreasing starch branching enzyme expression is responsible for the formation of heterogeneous starch granules. Plant Physiol 176:582-595

Wei C, Qin F, Zhu L, Zhou W, Chen Y, Wang Y, Gu M, Liu Q (2010) Microstructure and ultrastructure of high-amylose rice resistant starch granules modified by antisense RNA inhibition of starch branching enzyme. J Agric Food Chem 58: 1224-1232

Wilcox G (2005) Insulin and insulin resistance. Clin Biochem Rev 26:19-39

Yamamoto K, Sawada S, Onogaki T (1973) Properties of rice prepared by alkali method with various conditions. J Jpn Soc Starch Sci 20:99-104

Yamamoto K, Sawada S, Onogaki T (1981) Effects of quality and quantity of alkaline solution on the properties of the rice starch. J Jap Soc Starch Sci 28: 241-244

Zhou H, Wang L, Liu G, Meng $X$, Jing $Y$, Shu X, Kong $X$, Sun J, Yu H, Smith SM, Wu D, Li J (2016) Critical roles of soluble starch synthase SSIlla and granulebound starch synthase waxy in synthesizing resistant starch in rice. PNAS 113(45):12844-12849

Zhu L, Gu M, Meng X, Cheung SC, Yu H, Huang J, Sun Y, Shi Y, Liu Q (2012) High-amylose rice improves indices of animal health in normal and diabetic rats. Plant Biotechnol J 10:353-362

\section{Publisher's Note}

Springer Nature remains neutral with regard to jurisdictional claims in published maps and institutional affiliations.

\section{Submit your manuscript to a SpringerOpen ${ }^{\circ}$ journal and benefit from:}

- Convenient online submission

- Rigorous peer review

- Open access: articles freely available online

- High visibility within the field

- Retaining the copyright to your article

Submit your next manuscript at $\boldsymbol{\nabla}$ springeropen.com 